
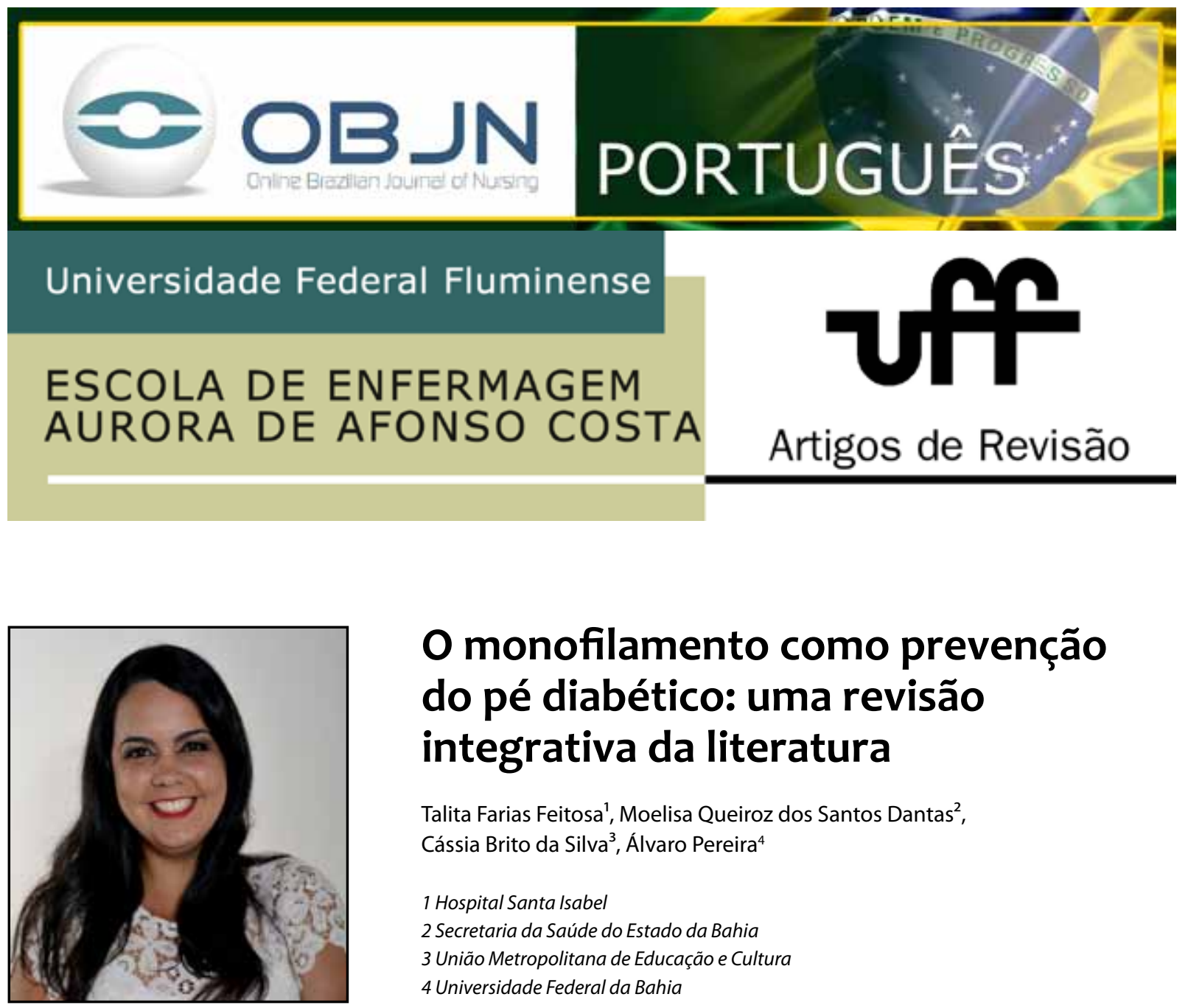

\title{
O monofilamento como prevenção do pé diabético: uma revisão integrativa da literatura
}

\author{
Talita Farias Feitosa', Moelisa Queiroz dos Santos Dantas², \\ Cássia Brito da Silva ${ }^{3}$, Álvaro Pereira ${ }^{4}$ \\ 1 Hospital Santa Isabel \\ 2 Secretaria da Saúde do Estado da Bahia \\ 3 União Metropolitana de Educação e Cultura \\ 4 Universidade Federal da Bahia
}

\section{RESUMO}

Objetivo: verificar, na produção científica, o grau de confiabilidade do monofilamento de SemmensWeinstein como um instrumento de avaliação do risco para o pé diabético. Método: trata-se de uma revisão integrativa da literatura realizada a partir de consulta nas bases de dados eletrônicas CINAHL, MEDLINE, SCOPUS e SCIELO. Resultados: foram selecionados seis artigos compreendendo cinco estudos transversais e um estudo de coorte. Os seis artigos incluídos na revisão são provenientes de revistas médicas, e não foi encontrada nenhuma publicação de enfermagem que atendesse ao objetivo. Conclusão: o monofilamento de Semmens-Weinstein é um instrumento confiável e que tem o melhor desempenho para a avaliação de risco para o pé diabético, sendo de extrema importância a sua aplicabilidade nas consultas prestadas.

Descritores: Pé Diabético; Neuropatias Periféricas; Medição de Risco; Cuidados de Enfermagem.

Feitosa TF, Dantas MQS, Silva CB, Pereira A. Monofilament for preventing the diabetic foot: an integrative review of the literature. Online braz j nurs [internet] 2016 Jun [cited year month day]; 15 (2):291-301. Available from: http://www.objnursing. uff.br/index.php/nursing/article/view/5277 


\section{INTRODUÇÃO}

O diabetes mellitus (DM) é uma doença crônica metabólica que é ocasionada pelo metabolismo anômalo dos hidratos de carbono, que resultam em defeitos na secreção insulínica, ocorrendo desta forma a elevação do nível de glicose no sangue ${ }^{(1)}$. Pode ser classificada em duas categorias, sendo a diabetes tipo I quando ocorre a falta de insulina no organismo, tornando necessária a utilização da insulinoterapia, e a diabetes tipo II caracterizada pela produção insuficiente de insulina, ou quando o organismo não consegue utilizá-la de forma eficaz ${ }^{(2)}$.

No Brasil, as doenças crônicas não transmissíveis correspondem a $72,4 \%$ das causas de mortes. Dados da Vigilância de Fatores de Risco e Proteção para Doenças Crônicas por Inquérito Telefônico (Vigitel) coletados em 2013 mostram que a prevalência de diabetes na população total é de $6,9 \%$ e que $12,2 \%$ da população diagnosticada, em ambos os sexos, possui até oito anos de escolaridade. Quando se compara a prevalência entre os sexos, observa-se que o diabetes acomete mais o sexo feminino $(7,2 \%)$ do que o masculino (6,5\%); além disso, nesse inquérito 8,5\% dos indivíduos com mais de 45 anos e $22,1 \%$ daqueles com mais de 65 anos referiram ser diabéticos ${ }^{(3)}$.

Dados obtidos pelo SisHiperdia (Sistema de Cadastramento e Acompanhamento de Hipertensos e Diabéticos) mostram que dentre os mais de 1,6 milhão de casos registrados de DM, 4,3\% tinham pé diabético e 2,2\% já fizeram amputações ${ }^{(4)}$. Vale ressaltar que cerca de $10 \mathrm{a}$ 25\% dos portadores de DM acima de 70 anos desenvolvem lesões nos membros inferiores; destes, 14 a 24\% tem o membro amputado(5).

Os membros inferiores são mais vulneráveis ao surgimento de ulcerações em pacientes portadores de DM. Desta forma, tem-se tra- balhado na necessidade de um cuidado mais especial com os pés desses pacientes. Mais de $10 \%$ dos pacientes diagnosticados com DM tem predisposição para o desenvolvimento de úlceras nos pés ${ }^{(6,7)}$.

O termo pé diabético é empregado nas alterações e complicações ocorridas nos pés dos pacientes com DM, que incluem a presença de infecção, ulceração e/ou destruição de tecidos profundos associados a anormalidades neurológicas e a vários graus de doença vascular periférica. Pode ser classificado em neuropático, vascular (isquêmico) e misto quando é neuropático e vascular ${ }^{(8)}$.

Os fatores de risco para o agravo do diabetes e o surgimento das úlceras nos pés são idade, tipo e tempo de diagnóstico, controle inadequado da glicemia, tabagismo, alcoolismo, obesidade, hipertensão, antecedente de úlcera nos pés, amputação não traumática, educação em saúde deficiente, neuropatia, calos e lesões não ulcerativas e uso de calçados inadequados ${ }^{(9)}$.

A presença da neuropatia periférica nos indivíduos diabéticos contribui para episódios de trauma e ulcerações, provocando a perda gradual da sensibilidade protetiva, da percepção da pressão plantar e da temperatura. A hipotrofia da musculatura intrínseca do pé, decorrente da neuropatia, pode desencadear deformidades osteoarticulares e alterações na marcha/deambulação, que também contribuem para o desenvolvimento das úlceras plantares ${ }^{(0,10)}$.

Um dos obstáculos para a prevenção do pé diabético é a falta da realização de exames dos pés nas consultas de rotina, embora essa já seja uma prática recomendada nos consensos nacionais e internacionais. $O$ enfermeiro possui o importante papel na orientação dos cuidados e na realização da consulta de enfermagem em pacientes portadores de DM, sendo também de sua responsabilidade o exame físico dos pés 
visando à prevenção do pé diabético ${ }^{(5)}$, pois sabe-se que $85 \%$ dos problemas relacionados ao pé diabético são susceptíveis a prevenção(10).

A avaliação neurológica dos pés pode ser realizada com a utilização das três técnicas recomendadas pelo Ministério da Saúde ${ }^{(8)}$ : avaliação da sensibilidade tátil com monofilamento de Semmens-Weinstem, avaliação da sensibilidade vibratória com diapasão de $128 \mathrm{~Hz}$ e avaliação do reflexo tendíneo Aquileu.

Embora o teste de monofilamento tenha sido originalmente utilizado na investigação de hanseníase, essa técnica tem demonstrado uma elevada especificidade no diagnóstico da neuropatia diabética ${ }^{(10,11)}$. Seu uso vem sendo indicado pela facilidade de realização do teste e boa relação custo-benefício, além da alta reprodutibilidade dos resultados e sua capacidade preditiva para ulcerações em diabéticos ${ }^{(11)}$.

O diagnóstico precoce da neuropatia periférica associada a medidas educativas e ao estímulo do autocuidado dos pés podem reduzir a incidência do pé diabético e o risco de amputações. Acredita-se que a utilização do teste do monofilamento Semmens-Weinstein na consulta de enfermagem tem um grande impacto nesse contexto.

Este estudo tem como objetivo geral verificar, na produção científica, o grau de confiabilidade do monofilamento de Semmens-Weinstein como um instrumento de avaliação do risco para o pé diabético, sendo atendido por meio dos seguintes objetivos específicos: verificar a existência de alternativas confiáveis à realização de um teste para a perda da sensibilidade dos pés, avaliando e discutindo o uso desses testes para a detecção precoce da neuropatia; avaliar a confiabilidade dos testes para detecção da neuropatia e comparar a funcionalidade de outros dispositivos ao monofilamento de Semmens-Weinstein.

\section{MÉTODO}

No presente estudo, a revisão integrativa da literatura é utilizada como método para a elaboração da pesquisa. Este método foi escolhido por propiciar a síntese de múltiplos estudos publicados, permitindo conclusões gerais, sendo mais amplo e tornando-se um método vantajoso por possibilitar a inclusão simultânea de pesquisas experimentais ou quase experimentais, oportunizando a formação de novos conhecimentos, baseados nos resultados apresentados por pesquisas anteriores ${ }^{(12)}$.

Com a intenção de melhorar os trabalhos de revisão integrativa da literatura, Souza, Silva e Carvalho ${ }^{(13)}$ listaram seis etapas do processo de elaboração da revisão integrativa, que foram seguidas neste trabalho: (1) elaboração da pergunta norteadora, (2) busca ou amostragem na literatura, (3) coleta de dados, (4) análise crítica dos estudos incluídos, (5) discussão dos resultados e (6) apresentação da revisão integrativa.

Para a elaboração da questão norteadora, utilizou-se a estratégia PICO (população de pacientes, intervenção, comparação e desfechos): o teste de monofilamento Semmens-Weinstein de $10 \mathrm{~g}$ é a melhor opção para a detecção precoce da neuropatia periférica em pacientes diabéticos?

Para a busca na literatura, foi escolhido o descritor pé diabético/diabetic foot (DeCS/ $\mathrm{MeSH}$ ) e a palavra-chave monofilamento. Foram considerados elegíveis os estudos disponíveis na íntegra, publicados nos últimos cinco anos (2010-2015) nos idiomas inglês, português e espanhol. Excluíram-se deste estudo artigos que falassem do uso do monofilamento para prevenção de outras patologias e sem determinação de metodologia clara.

O levantamento bibliográfico foi realizado nos meses de março e abril de 2015, por duas revisoras individualmente, utilizando o 
descritor pé diabético/diabetic foot sozinho e combinado à palavra-chave monofilamento com o operador boleano "and". A palavra-chave monofilamento também foi utilizada sozinha para a busca. Foram consultadas as bases de dados Cumulative Index to Nursing na Allied Helth Literature (CINAHL), Medical Literature Analysis and Retrivial System Online (MEDLINE), Sciverse Scopus e Scientific Eletronic Library Online (SCIELO). Os recursos disponíveis de cada base de dados para a recuperação de publicações foram utilizados para filtrar o tempo, o idioma e o tipo de estudo.

A relação obtida individualmente pelas revisoras foi composta pelas referências completas dos artigos, resumos e descritores. Cada revisor, durante a busca, aplicou os critérios de inclusão e exclusão previamente estabelecidos, realizou a leitura do título e do resumo. Para a obtenção da relação final, todos os resumos foram lidos por pelo menos dois revisores e, em casos de dúvidas sobre a inclusão de um estudo, o resumo foi lido por um terceiro revisor.

Todos os estudos selecionados a partir da leitura dos resumos foram obtidos e lidos na íntegra por pelo menos três revisores e em seguida foram explorados por meio do instrumento elaborado por URSI ${ }^{(13)}$ para a construção de revisões integrativas.

Souza, Silva e Carvalho (2010) recomendam a utilização do instrumento validado por URSI ${ }^{(13)}$, que contempla os seguintes aspectos: nome da pesquisa, tipo de publicação, detalhamento metodológico, detalhamento amostral, intervenção estudada, resultados, recomendações e conclusões. Este instrumento está disposto no Anexo A.

Os estudos foram analisados descritivamente e apresentados quanto ao idioma, país de origem, tipo de estudo, instrumento avaliado, nível de evidência, principais resultados encontrados.

\section{RESULTADOS}

O levantamento nas bases de dados geraram 59 artigos relacionados ao estudo. Após a aplicação dos critérios de inclusão e exclusão previamente definidos e análise dos títulos correlacionados com a temática, obteve-se uma amostra inicial com 24 artigos, que tiveram os resumos lidos e analisados conforme os objetivos da pesquisa, resultando na amostra final de seis artigos.

Tabela 1 - Distribuição dos artigos segundo o idioma. Lauro de Freitas, 2015.

\begin{tabular}{lcc}
\hline \multirow{2}{*}{ IDIOMA } & \multicolumn{2}{c}{ FREQUÊNCIA } \\
\cline { 2 - 3 } & $\mathbf{N}^{\circ}$ & $\%$ \\
\hline Inglês & 5 & 84 \\
Português & 1 & 16 \\
TOTAL & 6 & 100 \\
\hline
\end{tabular}

Fonte: elaborada pelos autores.

Para a coleta de dados, os artigos selecionados na amostra final foram enumerados de um (1) a seis (6), de acordo com o ano de publicação, de forma crescente. Estes estão identificados, apresentando a referência bibliográfica disposta no Quadro 01:

A amostra compreendeu cinco estudos transversais e um estudo de coorte. É importante ressaltar que os seis artigos incluídos na revisão são provenientes de revistas médicas, não sendo identificadas publicações em revistas de enfermagem.

Os artigos selecionados estavam escritos em dois idiomas, inglês e português, seguindo os critérios de inclusão do estudo. Conforme se pode notar na Tabela 1 um, 84\% dos artigos foram escritos em inglês e $16 \%$ dos artigos em português.

A partir dos dados apresentados acima e com o objetivo de caracterizar a amostra, foram descritos os dados referentes à identificação dos países de origem dos artigos, apresentados na Tabela 2. 
Figura 1 - Fluxograma explicativo da seleção os artigos para formar a amostra. Lauro de Freitas, 2015.

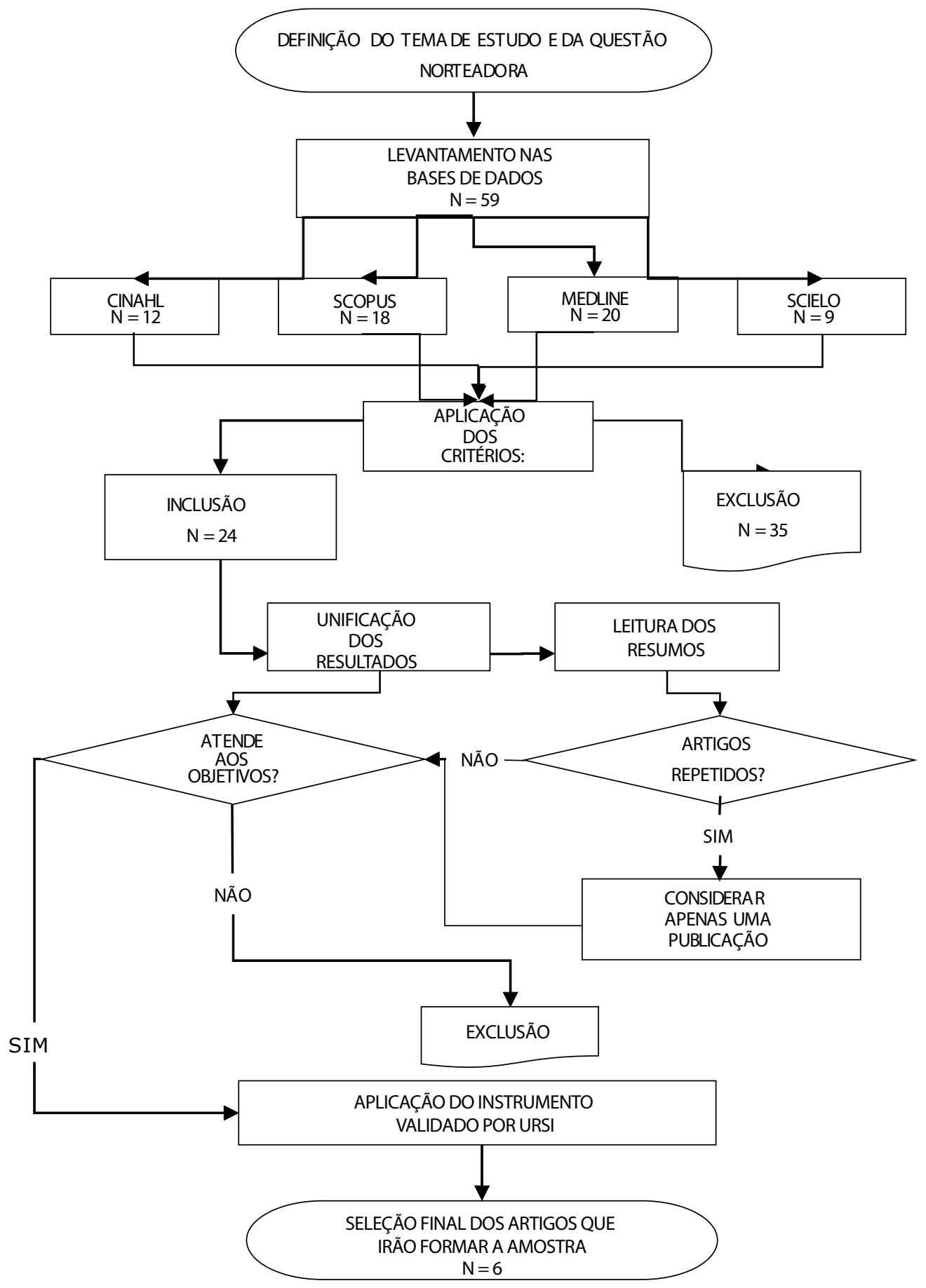

Fonte: elaborada pelos autores.[FIGURA 1]

Feitosa TF, Dantas MQS, Silva CB, Pereira A. Monofilament for preventing the diabetic foot: an integrative review of the literature. Online braz j nurs [internet] 2016 Jun [cited year month day]; 15 (2):291-301. Available from: http://www.objnursing. uff.br/index.php/nursing/article/view/5277 
Quadro 1 - Relação dos artigos que fizeram parte da amostra. Lauro de Freitas, 2015.

\begin{tabular}{|c|l|}
\hline 1 & $\begin{array}{l}\text { COLLINS, S., VISSCHER, P., VET, H. C. DE, ZUURMOND, W. W. A., PEREZ, R. S. G. M. Reability of the Semmes Weinstein } \\
\text { Monofilaments to measure coetaneous sensibility in the feet of healthy subjects. Disability and Rehabilitation, } \\
\text { Amsterdam, v. 32, n. 24. P. 71-74. 2010. }\end{array}$ \\
\hline 2 & $\begin{array}{l}\text { FERREIRA, M. C., VIEIRA, S. A. T., de CARVALHO, V. F. Estudo comparativo da sensibilidade nos pés de diabéticos } \\
\text { com e sem úlceras utilizando o PSSD. ACTA Ortopédica Brasileira, v. 18, n. 02. P. 71-74. } 2011 .\end{array}$ \\
\hline 3 & $\begin{array}{l}\text { ELLAWAY, P. H., CATLEY, M. Reliability of the electrical perceptual thereshold and Semmes-Weinstein monofila- } \\
\text { ment test of cutaneous sensibility. Spinal Cord, v. 51, n. 02. P. 120-125. Fevereiro. 2013. }\end{array}$ \\
\hline 4 & $\begin{array}{l}\text { KATON, J. G., REIBER, G. E., NELSON, K. M. Peripheral Neuropathy Defined by Monofilament Insensitivity and Diabe- } \\
\text { tes Status. Diabetes Care, v. 36, n. 06. P. 1604-1606. Junho. 2013. }\end{array}$ \\
\hline 5 & $\begin{array}{l}\text { SLATER, R. A., KOREN, S., RAMOT, Y., BUCHS, ANDREAS., RAPOPORT, M. J. Interpreting the results of the Semmens- } \\
\text {-Weinstein monofilament test: accounting for false-positive answers in the international consensus on the diabe- } \\
\text { tic foot protocol by a new model. Diabetes Metabolism Research and reviews, v. 30, n. 01. P. 77-80. Janeiro. 2014. }\end{array}$ \\
\hline 6 & $\begin{array}{l}\text { HIRE, J. M., RAMADORAI, U. E., CONTRACTOR, S., JACOBS, J. M., BOJESCUL, J. A., ABELL, B. E. Intravenous Angio- } \\
\text { catheters as a Novel Alternative to Semmes-Weinstein Monofilament Evaluation in Testing Protective Sensation. } \\
\text { Military Medicine, v. 179, n. 04. P. 442-444. Abril. 2014. }\end{array}$ \\
\hline
\end{tabular}

Fonte: elaborado pelos autores.

Quadro 2 - Equipamentos/instrumentos utilizados como diagnóstico da neuropatia periférica. Lauro de Freitas, 2015.

\begin{tabular}{|c|l|l|l|l|}
\hline ARTIGO & TIPO & AUTOR & ANO & EQUIPAMENTOS/INSTRUMENTOS \\
\hline 1 & Estudo Transversal & $\begin{array}{l}\text { COLLINS, S., VISSCHER, P., } \\
\text { VET, H. C. DE, ZUURMOND, } \\
\text { W. W. A., PEREZ, R. S. G. M. }\end{array}$ & 2010 & Semmes-Weinstein \\
\hline 2 & Estudo Transversal & $\begin{array}{l}\text { FERREIRA, M. C., VIEIRA, S. A. } \\
\text { T., de CARVALHO, V. F. }\end{array}$ & 2010 & PSSD \\
\hline 3 & Estudo Transversal & ELLAWAY, P. H., CATLEY, M. & 2012 & $\begin{array}{l}\text { Teste do Limiar de Percepção Elétri- } \\
\text { ca e Semmens-Weinstein }\end{array}$ \\
\hline 5 & $\begin{array}{l}\text { Estudo de Coorte Retros- } \\
\text { pectivo }\end{array}$ & $\begin{array}{l}\text { KATON, J. G., REIBER, G. E., } \\
\text { NELSON, K. M. }\end{array}$ & 2013 & Semmens-Weinstein \\
\hline 6 & Estudo Transversal & $\begin{array}{l}\text { SLATER, R. A., KOREN, S., RA- } \\
\text { MOT, Y., BUCHS, ANDREAS., } \\
\text { RAPOPORT, M. J. }\end{array}$ & 2014 & Semmens-Weinstein \\
\hline
\end{tabular}

Fonte: elaborada pelos autores.

Tabela 2 - Distribuição dos estudos segundo o país de origem do artigo. Lauro de Freitas, 2015.

\begin{tabular}{lcc}
\hline \multicolumn{1}{c}{ PAÍS } & No $^{\circ}$ & $\%$ \\
\hline EUA & 2 & 32 \\
Brasil & 1 & 17 \\
Holanda & 1 & 17 \\
Israel & 1 & 17 \\
Reino Unido & 1 & 17 \\
TOTAL & 6 & 100 \\
\hline
\end{tabular}

Fonte: elaborada pelos autores.
Os Estados Unidos foram o país que mais produziu artigos com o tema proposto neste estudo. Percebe-se a escassez de estudos publicados que utilizam o monofilamento para detecção precoce da neuropatia periférica. No entanto, até hoje não se dispõe de publicações nacionais sobre confiabilidade do teste de Semmens-Weinstein com a finalidade de detectar precocemente a neuropatia periférica. De acordo com os resultados, pode-se notar que $84 \%$ das amostras são de origem estrangeira.

Feitosa TF, Dantas MQS, Silva CB, Pereira A. Monofilament for preventing the diabetic foot: an integrative review of the literature. Online braz j nurs [internet] 2016 Jun [cited year month day]; 15 (2):291-301. Available from: http://www.objnursing. uff.br/index.php/nursing/article/view/5277 
O Quadro 2 mostra os diferentes tipos de métodos utilizados e testados como possíveis instrumentos para o exame clínico na detecção precoce da neuropatia periférica.

Quanto ao objeto, três artigos utilizaram o monofilamento como teste para o diagnóstico de perda da sensibilidade periférica, um artigo empregou a técnica de PSSD (Pressure-Specified Sensory Device), um usou o Teste do Limiar de Percepção Elétrica para comparar seus resultados ao monofilamento de Semmens-Weinstein a fim de comparar a confiabilidade dos testes e um utilizou o angiocatéter como equipamento para testar a sensibilidade periférica como possível substituto ao monofilamento.

O artigo 01 objetiva determinar a confiabilidade intra-avaliador, interobservador de confiabilidade e de pontuação de referência normal no teste de Semmens-Weinstein dos pés de indivíduos saudáveis. Concluiu-se que as medições do monofilamento de Semmens-Weinstein (SW) do lado plantar dos pés são confiáveis quando medidas por um único pesquisador. O SW é capaz de detectar alterações em verdadeiros limites sensoriais, no entanto não fica claro se o monofilamento de SW é suficientemente estável para ser utilizado em pesquisas.

O artigo 02 determina e compara os limiares de sensibilidade cutânea nos pés de pacientes diabéticos com úlcera em apenas um dos membros inferiores. Ficou claro que o PSSD pode ser mais preciso do que o teste com o monofilamento de SW, pois este não diferencia os membros com e sem úlceras como o PSSD, que é um equipamento confiável e útil para realizar diagnóstico preciso da perda de sensibilidade em pacientes diabéticos de maneira quantitativa demonstrável.

O artigo 03 compara a confiabilidade dos testes de Limiar de Percepção Elétrica (EPT) e
SW da sensibilidade cutânea em uma população neurologicamente saudável, tendo como resultado pequenas alterações nos limites de 95\% entre os diferentes dermátomos para ambos os métodos, não havendo relação entre o tamanho da diferença, as avaliações e a magnitude média de uma medida para qualquer dermátomo por qualquer um dos métodos.

$\mathrm{O}$ artigo 04 determina se o status do diabetes está associado ao aumento do risco de neuropatia periférica utilizando o monofilamento de SW como teste de sensibilidade, cujos resultados sugerem que o DM esteja associado a um risco quase duas vezes maior para neuropatia periférica.

0 artigo 05 estuda o significado, até então não examinado, de respostas falso-positivas. Fica comprovado que respostas falso-positivas no teste de SW são comuns em pacientes diabéticos com e sem história de ulceração e pode ser um fator importante na avaliação dos resultados.

O artigo 06 testa a funcionalidade do angiocateter para substituir o monofilamento de SW, em caso de indisponibilidade. Concluiu-se que o angiocatéter pode complementar a gama de ferramentas para os exames prestados por cuidados de saúde, no entanto, no padrão ouro permanece o SW de $10 \mathrm{~g}$.

\section{DISCUSSÃO}

Diferentes estudos têm sido realizados sobre o pé diabético. É possível observar que o método mais testado ainda é o monofilamento de Semmens-Weinstein, com o qual se pode concluir que as medições são confiáveis quando aferidas por um único pesquisador. Além disso, a pontuação sensorial normal situa-se entre 3,22 e 4,08 $8^{(14)}$, sendo este confiável e de fácil manuseio, porém pesquisas recentes 
mostram a apreensão em encontrar outra técnica para substituir o monofilamento em caso de falta, ou que possa ser mais seguro, e também a preocupação com a confiabilidade dos resultados de monofilamento de SW como prevenção do pé diabético.

A análise comparativa com os testes de EPT e de SW como sendo dois testes que podem avaliar diferentes modalidades de sensibilidade cutânea mostra que existem pequenas diferenças dentro de um limite de 95\% entre si. O ICC (Coeficiente de Correlação Interclasses) variou entre $0,46-0,61$ para o teste de Semmens-Weinstein e 0,67-0,81 para o $E P T$, concluindo que o indivíduo em risco se beneficiaria ao realizar os dois exames, visto que um complementa o outro ${ }^{(15)}$.

Há cada vez mais tratamentos e exames sofisticados para combater o impacto do diabetes. O não cumprimento destes na prevenção precoce impacta na qualidade de vida dos portadores ${ }^{(16)}$. Quanto aos equipamentos/instrumentos que foram utilizados de maneira alternativa ao monofilamento de SW nos estudos, destacou-se que o PSSD, por ser um equipamento mais preciso e capaz de diferenciar os membros com e sem lesão, é um possível exame complementar ao teste de SW a ser realizado na triagem para avaliar a perda da sensibilidade, já que o valor de $p$ foi menor que $0,001 \mathrm{em}$ todos os testes realizados com este equipamento, demonstrando significância estatística ${ }^{(17)}$. Também foi identificado que o Angiocatéter de $24 \mathrm{~g} \times 0,75$ pode substituir o monofilamento de SW em caso de falta ou verba para adquiri-lo, na realização do exame e detecção precoce do risco para o pé diabético, tendo uma confiabilidade com valores superiores a 0,98 ( $p<0,001)$. No entanto, o padrão ouro permanece o monofilamento de SW, com a sensibilidade relatada de até 0,95 e especificidade de até 0,82 para o diagnóstico de perda da sensibilidade, comprovando a sua eficácia e confiabilidade $\mathrm{e}^{(18,19,20)}$.

Almeja-se a intensificação da utilização de avaliação sistemática dos indivíduos juntamente com o teste de monofilamento de SW, objetivando a manutenção das medidas preventivas quanto às complicações. $\mathrm{O}$ uso do teste de SW seria suficiente para o diagnóstico do paciente em risco de neuropatia durante as consultas de rotina da atenção básica. Os indivíduos em risco devem entender as implicações da perda da sensação protetora e a importância de ter o cuidado diário com os pés ${ }^{(6,9,21)}$.

Uma vez que um paciente perde a sensibilidade ao monofilamento de SW, ainda não foram encontrados estudos que falam sobre o acompanhamento dado a este individuo quanto à prevenção e/ou tratamentos pres$\operatorname{tados}^{(22)}$.

Este estudo reforça a necessidade de unidades para o tratamento especializado de pacientes com diabetes, onde o risco para desenvolver a neuropatia periférica é valorizado; cria um sistema para classificar o risco e/ ou a evolução do pé diabético; locais em que se possa planejar e executar programas de prevenção, detecção precoce dessas doenças, a fim de evitar as frequentes amputações de membros inferiores ${ }^{(23)}$.

\section{CONCLUSÃO}

Os resultados deste estudo possibilitaram alcançar os objetivos propostos, concluindo que o teste de monofilamento de SW é confiável para a detecção precoce do pé diabético, sendo de extrema importância a sua aplicabilidade nas consultas prestadas a pacientes diabéticos, podendo, ainda, ser complementado com outros exames, como o PSSD, a fim 
de se obter um resultado ainda mais especifico quanto à existência da neuropatia periférica.

$O$ estudo permitiu o conhecimento de novos equipamentos como o PSSD e o Angiocatéter $24 \mathrm{~g} X 0,75$, que auxiliam o monofilamento de SW de forma confiável, podendo ser realizado em conjunto, ou até mesmo substituindo o monofilamento e SW.

Embora tenha sido testada e aprovada a confiabilidade do teste de SW para a detecção precoce da neuropatia periférica, a sua aplicabilidade ainda tem sido negligenciada dado ao crescente diagnóstico de pé diabético, que pode ser comprovado por meio do alto índice de pé diabético e de amputações dos membros inferiores por consequência da neuropatia periférica.

O enfermeiro, além de estimular o cuidado com os pés e promover ações educativas sobre os agravos do diabetes, deve examinar e aplicar o teste monofilamento de SW, pois ele continua sendo o padrão ouro, por ser de baixo custo e fácil manuseio, confiável, visando contribuir para a prevenção da neuropatia periférica e suas consequências, possibilitando sensibilizar estes indivíduos para o desenvolvimento do autocuidado e promoção da qualidade de vida.

Embora o monofilamento de SW tenha sido potencialmente capaz de identificar o risco para o pé diabético, novos estudos devem ser realizados a fim de se conhecer o impacto do diagnóstico precoce da neuropatia periférica na prevenção do pé diabético, considerando o cenário atual das políticas públicas brasileiras de saúde. Nesse contexto, é necessário conhecer como se prediz esse risco quando se considera o uso do monofilamento SW individualmente ou combinado com outras técnicas, como a exemplo do PSSD, desde a primeira consulta na rede básica de saúde.

\section{REFERÊNCIAS}

1. Martin VT, Rodrigues CDS, Cesarino CB. Conhecimento do paciente com diabetes mellitus sobre o cuidado com os pés. Rev Enferm UERJ [periodic online]. 2011 Oct/Dec [Cited 2014 May 12]; 19(4):621-5. Available from: http:// www.facenf.uerj.br/v19n4/v19n4a20.pdf.

2. Martins PAG. Diabetes Mellitus: novas abordagens terapêuticas [tese online]. Capariga: Instituto Superior de Ciências da Saúde Egas Moniz; 2015 [Cited 2016 May 3]. Available from: http:// comum.rcaap.pt/bitstream/10400.26/10951/1/ Martins\%2c\%20Patr\%C3\%ADcia\%20Alexandra\%20Guerreiro.pdf

3. Ministério da Saúde (Brasil), Secretária de Vigilância em Saúde, Coordenação Geral de Doenças e Agravos não transmissíveis. Vigitel Brasil 2013. Brasília: Ministério da Saúde; 2014 [Cited 2015 June 5]. Available from: http://www.prefeitura.sp.gov.br/cidade/secretarias/upload/ saude/arquivos/morbidade/Vigitel-2013.pdf.

4. Schmidt MI, Duncan BB, Silva GA, Menezes AM, Monteiro CA, Barreto SM, et al. Chronic non-communicable diseases in Brazil: burden and current challenges. The Lancet [periodic online]. 2011 May [Cited 2014 Jun 8]; 377(9781):60135-9. Available from: http:// www.sbh.org.br/pdf/lancet_collection.pdf.

5. Cubas MR, Santos OM, Retzlaff EMA, Telma HLC, Andrade IPS, Moser ADL, et al. Pé diabético: orientações e conhecimento sobre cuidados preventivos. Fisioter mov [periodic online]. $2013 \mathrm{Jul} / \mathrm{Set}$ [Cited 2014 May 12]; 26(3):647-55. Available from: http://www.scielo.br/pdf/fm/ v26n3/a19v26n3.pdf.

6. Andrade NHS, Mendes KDS, Faria HTG, Martins TA, Santos MA, Teixeira CRS, et al. Paciente com Diabetes Mellitus: cuidados e prevenção do pé diabético em atenção primária à saúde. Rev Enferm UERJ [periodic online]. 2010 Oct/ Dec [Cited 2014 May 12]; 18(4):616-21. Available from: http://www.facenf.uerj.br/v18n4/ v18n4a19.pdf.

7. Caiafa JS, Castro AA, Fidelis C, Santos VP, Silva ES, Junior Sitrângulo CJ. Atenção integral ao portador de pé diabético. J Vasa Bras [periodic 
online]. 2011 [Cited May 12]; 10(4 Suppl 2): 1-32. Available from: http://www.scielo.br/pdf/ jvb/v10n4s2/a01v10n4s2.pdf.

8. Ministério da Saúde (Brasil), Secretaria de Atenção à Saúde, Departamento de Atenção Básica. Manual do pé diabético: estratégias para o cuidado da pessoa com doença crônica. Brasília: Ministério da Saúde, 2016 [Cited 2016, May 03]. Available from: http://189.28.128.100/ dab/docs/portaldab/publicacoes/manual_do_ pe_diabetico.pdf.

9. Santos ICRV, Bezerra GC, Souza CL, Pereira LC, et al. Pé diabético: apresentação clínica e relação com o atendimento na atenção básica. Rev Rene [periodic online]. 2011 Apr/Jun. [Cited 2014 May 12]; 12(2):393-400. Available from: http://www.revistarene.ufc.br/vol12n2_pdf/ a22v12n2.pdf.

10. Mendonça SS, Morais JS, Moura MCGG. Proposta de um protocolo de avaliação fisioterapêutica para os pés de diabéticos. Fisioter mov [periodic online]. 2011 Apr/June [Cited 2014 May 12]; 24(2):285-98. Available from: http:// www.scielo.br/pdf/fm/v24n2/a10v24n2.

11. Schmidt MI, Melere C, Damé P. Manual de Procedimento: monofilamento. ELSA-BRASIL. 2012. [S.I.: s.n.].

12. Botelho LLR, Cunha CCA, Macedo M. O método da revisão integrativa nos estudos organizacionais. Gestão e Sociedade [periodic online]. 2011 May/Aug [Cited 2014 Jun 6]; 5(11):121-136. Available from: http://www.gestaoesociedade.org/gestaoesociedade/article/ view/1220/906.

13. Souza MT de, Silva MD da, Carvalho R de. Revisão Integrativa: o que é e como fazer. Einstein [periodic online]. 2010 Jan/Mar [Cited 2014 Jun 6]; 8(1):102-6. Available from: http://www.astresmetodologias.com/material/O_que_e_RIL. pdf.

14. Collins S, Visscher P, Vet HC de, Zuurmond WWA, Perez RSGM. Reability of the Semmes Weinstein Monofilaments to measure coetaneous sensibility in the feet of healthy subjects. Disabil Rehabil. 2010; 32(24):71-4. [included in the review]

15. Ellaway PH, Catley M. Reliability of the electrical perceptual thereshold and Semmes-Weinstein monofilament test of cutaneous sensibility. Spinal Cord. 2013; 51(2):120-5. [included in the review]

16. Talarico R. Preventing diabetic foot amputations: podiatry, protocols and perfusion. Podiatry Management. 2013 nov./dec; 32(9):127-134.

17. Ferreira MC, Vieira SAT, de Carvalho VF. Estudo comparativo da sensibilidade nos pés de diabéticos com e sem úlceras utilizando o PSSD. Acta Ortop Bras. 2011; 18(2):71-4. [included in the review]

18. Katon JG, Reiber GE, Nelson KM. Peripheral Neuropathy Defined by Monofilament Insensitivity and Diabetes Status. Diabetes Care. 2013 Jun; 36(6):1604-6. [included in the review]

19. Slater RA, Koren S, Ramot $Y$, Buchs A, Rapoport MJ. Interpreting the results of the Semmens-Weinstein monofilament test: accounting for false-positive answers in the international consensus on the diabetic foot protocol by a new model. Diabetes Metab Res Rev. 2014 Jan; 30(1):77-80. [included in the review]

20. Hire JM, Ramadorai UE, Contractor S, Jacobs JM, Bojescul JA, Abell BE. Intravenous Angiocatheters as a Novel Alternative to Semmes-Weinstein Monofilament Evaluation in Testing Protective Sensation. Mil Med. 2014 Apr; 179(4):442-4. [included in the review]

21. Santos ICRV, Nunes ENS, Melo CA, Farias DG. Amputações por pé diabético e fatores sociais: implicações para cuidados preventivos de enfermagem. Rev Rene [periodic online]. 2011 Oct/Dec [Cited 2014 may 12]; 12(4):68491. Available from: http://www.revistarene.ufc. br/revista/index.php/revista/article/view/283/ pdf.

22. Arad Y, Peters A, Fonseca V, Vinik A. Beyond the monofilament for the insensate diabetic foot. Diabetes Care. 2011 Apr; 34(4):1041-6.

23. Torre HGL, Fernández AM, Lorenzo MLQ, Pérez EP, Montesdeoca MPQ. Clasificaciones de lesiones en pie diabético. Um problema no resuelto. Gerokomos [periodic online]. 2012 June [Cited 2014 May 12]; 23(2):75-87. Available from: http://scielo.isciii.es/pdf/geroko/ v23n2/helcos1.pdf. 
Todos os autores participaram das fases dessa publicação em uma ou mais etapas a seguir, de acordo com as recomendações do International Committe of Medical Journal Editors (ICMJE, 2013): (a) participação substancial na concepção ou confecção do manuscrito ou da coleta, análise ou interpretação dos dados; (b) elaboração do trabalho ou realização de revisão crítica do conteúdo intelectual; (c) aprovação da versão submetida. Todos os autores declaram para os devidos fins que são de suas responsabilidades o conteúdo relacionado a todos os aspectos do manuscrito submetido ao OBJN. Garantem que as questões relacionadas com a exatidão ou integridade de qualquer parte do artigo foram devidamente investigadas e resolvidas. Eximindo, portanto o OBJN de qualquer participação solidária em eventuais imbróglios sobre a materia em apreço. Todos os autores declaram que não possuem conflito de interesses, seja de ordem financeira ou de relacionamento, que influencie a redação e/ou interpretação dos achados. Essa declaração foi assinada digitalmente por todos os autores conforme recomendação do ICMJE, cujo modelo está disponível em http://www. objnursing.uff.br/normas/DUDE_final_13-06-2013.pdf
Recebido: 03/08/2015

Revisado: 27/04/2016

Aprovado: $27 / 04 / 2016$ 